\title{
Generalized Space-Time Super-Modulation for Headerless Grant-Free Rateless Multiple Access
}

\author{
Farhad Mehran and Konstantinos Nikitopoulos \\ 5G Innovation Centre \\ Institute for Communication Systems \\ University of Surrey, Guildford, UK \\ Email: \{f.mehran, k.nikitopoulos\}@ surrey.ac.uk
}

\begin{abstract}
This work introduces Generalized Space-Time Super-Modulation (GSTSM), a generalization of the recently proposed Space-Time Super-Modulation scheme that enables the transmission of additional, highly-reliable information on the top of conventionally transmitted symbols, without increasing the corresponding packet length. GSTSM jointly exploits the spatial and temporal dimensions of multiple-antenna systems but, in contrast to the initially proposed approach, it does not require the use of space-time block codes. Instead, GSTSM jointly elaborates on the concepts of spatial modulation and spatial diversity, while intentionally introducing temporal correlation to the transmitted symbol sequence. In the context of machine-type communications, GSTSM enables one-shot and grant-free medium access without transmitting additional headers to convey each machine's ID. As a result, we show that GSTSM can provide throughput gains of up to $2.5 \times$ compared to conventional header-based schemes, even in the case of colliding packets.
\end{abstract}

Index Terms-Multiple-input multiple-output (MIMO), Grantfree access, Spatial modulation, Multilevel codes, Machine-type communications (MTCs).

\section{INTRODUCTION}

Machine Type Communications (MTCs) are foreseen as a major source of traffic in future wireless systems [1]. In real-world scenarios, MTC traffic is expected to be sporadic, uplink-centric, and to consist of short packet transmissions from a massive number of devices [2]. Current access control methods are not suitable for MTCs due to their high signaling overhead and latency [3]. For example, uplink access procedure of LTE Random Access Channel (RACH) requires approximately 59 bytes of overhead for transmission of 100 bytes of data [4]. As a remedy, grant-free access methods have been proposed where the users can transmit their information without sending scheduling requests [5]. Such grant-free methods are also referred to as "joint medium access and data" or "one-shot" transmissions.

Ideally, for maximizing the achievable throughput and reliability, as well as for minimizing the latency of future oneshot MTCs, the receiver should reliably identify the machine's identity (ID) even if the transmitted packet is lost. This will allow to combine undecoded packets of the same machine, in order to improve decoding efficiency. For reliably identifying each machine's ID, the corresponding traditionally transmitted packet header should be very well protected. This can be achieved either by transmitting long orthogonal sequences or by transmitting long and strongly (channel) encoded headers
[6]. After reliably identifying the transmitting machine, to efficiently combine its undecoded packets at the receiver side, rateless coding on the physical (PHY) layer can be used [7]. Still, to recover ratelessly encoded packets, besides the machine's ID, the receiver needs to know the ordered position of the received packet in the sequence of rateless coded packets. This requires additional signaling and, in traditional systems, it results in heavier header overhead.

To alleviate the need for transmitting headers, to the best of the authors' knowledge, the only solution that has been proposed so far is Space-Time Super Modulation (STSM) [8],[9]. STSM enables the transmission of additional information on the top of conventional information by exploiting the redundancy of space-time block codes [10]. This enables the encoding of the packet's signature information (SI) on the top of ratelessly encoded blocks, and therefore it obviates the need for transmitting packet headers or preambles. The focus of the current work is to generalize the concept of STSM to MIMO systems that are not space-time block encoded, and to arbitrarily large numbers of transmitter/receiver antennae. In this direction, GSTSM jointly exploits the spatial and temporal dimensions of multiple-antenna systems by developing the ideas of spatial modulation [11] and receive diversity of large MIMO systems [12] while intentionally introducing temporal correlation to the transmitted sequence. In contrast to the traditional spatial modulation [13], GSTSM enables the transmission of highly-reliable additional information with a flexible rate, and can also efficiently operate even in highly correlated channel conditions, or even in Additive White Gaussian Noise (AWGN) channels. Also, while spatial modulation explores on the spatial dimension, GSTSM explores both the spatial and temporal dimensions. As a result, spatially modulated systems require a significantly higher number of transmit antennae to transmit the same amount of additional information.

Since GSTSM is based on increasing the Euclidean distance between possible codewords to convey the additional transmitted information, it can be considered to be a member of Multi-Level Codes (MLC) family [14]. Unlike traditional MLCs [15], however, the information streams are not coded by individual co-optimized codes. Instead, GSTSM encodes the additional information by alternating the transmission patterns of conventional information and, therefore, GSTSM can be used with various types of channel codes. Also, other 
MLCs including Trellis Coded Modulation [16] and its spatial modulated versions such as Trellis Coded Spatial Modulation [17] target the minimization of the error-rate performance by joint optimization of modulation and coding, and are only based on convolutional codes. In contrast, GSTSM supports an additional information subchannel of flexible rate and is independent of the choice of the channel coding scheme.

To the best of our knowledge, GSTSM is the first approach that enables the transmission of additional information with flexible rate and reliability, by exploiting both the spatial and temporal dimensions of MIMO systems and in the absence of space-time block coding. Also, it is the first time that such a transmission scheme is utilized in the context of machine-type communications for enabling uncoordinated, grant-free, and headerless transmission of ratelessly encoded data packets.

\section{Generalized Space-Time Super-Modulation (GSTSM)}

The encoding and decoding procedures of GSTSM are presented in this section. As it will be shown, GSTSM can be adopted by systems with a various number of transmitting antennae and an arbitrarily large number of receiving antennae to exploit receive diversity. However, as for MTCs a low number of transmitting antennae is expected, we herein focus on systems with two transmit antennae and a single RF chain. Also, though various density of constellations [18] can be used in GSTSM, we focus on systems with BPSK as we target MTC scenarios with unfavorable transmission conditions.

\section{A. GSTSM Encoding}

GSTSM encodes the additional information on the top of conventional information, by altering the transmission patterns. This is achieved by elaborating on the ideas of spatial modulation [11] and receive diversity of large MIMO systems [12] and by introducing temporal correlation to the transmitted symbol sequences. Similarly to spatial modulation, GSTSM exploits the different propagation characteristics of the different transmitting antennae. However, rather than allocating distinct bit sequences to each transmitting antenna, GSTSM employs two-dimensional transmission patterns. Then, the additional information is conveyed by mapping the additional information sequence onto these patterns that define how the signal transmission takes place. In particular, one dimension of the pattern is related to the spatial dimension of the system, and dictates which of the transmitting antennas will be used (i.e., activated) for signal transmission. The other dimension, is related to the temporal characteristics of the signal and dictates how the conventionally transmitted information will be phase-rotated. As we show in Section IV, the existence of multiple-antennas at the receiver side enables extremely reliable detection of the corresponding patterns (and therefore of the super-modulated information).

In GSTSM systems with two transmit antennae, only one transmit antenna is activated for data transmission at any time instant. Based on the additional information bits, a pattern is chosen which determines the phase rotations and indices of

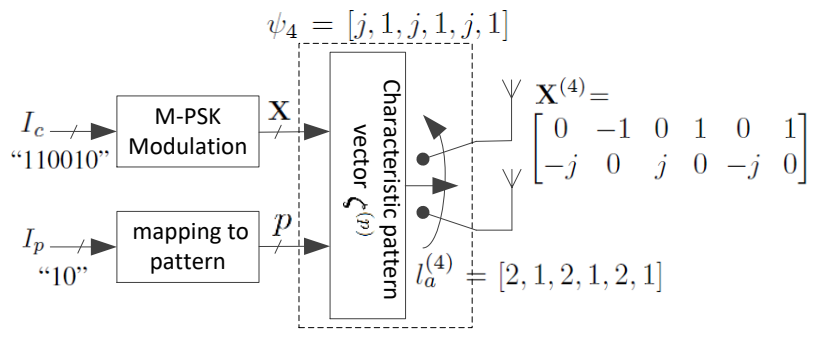

Fig. 1: GSTSM encoder with characteristic pattern vector $\boldsymbol{\zeta}^{(p)}$, employing two transmitting antennae with one RF chain. The numerical representations are regarding the given example.

the transmitting antennae for the transmission of conventional information symbols. Fig. 1 depicts the block diagram of the encoder. The transmitted information for each GSTSM information block is divided into two subsets. The first subset, $I_{c}$, consists of the conventionally modulated information bits, which are bits that are mapped onto a conventional constellation and would have been transmitted even in the absence of GSTSM. Considering the transmission of conventionally modulated bits of a block length of $K$ symbols, $K \log _{2}|\mathcal{M}|$ bits can be mapped onto conventional complex information symbols $x_{i}$, drawn from a complex constellation $\mathcal{M}$ of size $|\mathcal{M}|$ where $i=1, \ldots, K$. Hence, the conventionally modulated word is given by

$$
\mathbf{X}=\left[\begin{array}{lll}
x_{1} & \ldots & x_{K}
\end{array}\right]
$$

The second subset $I_{p}$ with a block length of $n_{p}$ bits, contains the super-modulated bits (i.e., the additional information) that are going to be encoded by means of GSTSM. The information bits of this stream are mapped onto a pattern $p$ via an appropriate bit-to-symbol mapping. Each pattern $p$ is related to its corresponding characteristic pattern vector $\zeta^{(p)}$ which describes how the conventionally modulated symbols will be super-modulated. To generate the super-modulated words, it is assumed that each conventional symbol can be further modulated by using one of the $\mathcal{O}_{s m}$ prespecified super-modulated states, that consist of prespecified phase rotations and active antenna indices. Hence, the characteristic pattern vector is defined as $\boldsymbol{\zeta}^{(p)}:=\left(\boldsymbol{\Lambda}^{(p)}, \boldsymbol{\xi}^{(p)}\right)$, where $\boldsymbol{\Lambda}^{(p)}$ and $\boldsymbol{\xi}^{(p)}$ are characteristic antennae activation and phase rotation vectors, respectively. For the conventionally modulated bits with symmetric PSK of size $|\mathcal{M}|$ and $\mathcal{O}$ available phase rotation states, the phase rotations can be $\psi_{p, k}=\exp \left\{j \frac{2 \pi}{\mathcal{O}|\mathcal{M}|}\left(\boldsymbol{\xi}_{k}^{(p)}-1\right)\right\}$. Here, the characteristic phase rotation vector $\boldsymbol{\xi}_{k}^{(p)}$ specifies the phase rotation state of pattern $p$ at time instant $k$. The phase rotated conventionally modulated symbol is denoted by $\grave{x}_{k}=x_{k} \psi_{p, k}$. In order to make the patterns distinguishable at the receiver, the phase rotations for all possible patterns are designed such that the rotated symbols do not coincide for any conventionally transmitted constellation point. These phase rotations also boost the detectability of the additional information in the case of correlated channel conditions. 
Subsequently, the phase rotated symbols are transmitted from the antenna that is specified by the characteristic antenna activation vector $\boldsymbol{\Lambda}^{(p)}$. The index of the active antenna is denoted by $l_{a}^{(p)} \in\left\{1, \ldots, N_{t}\right\}$, and the phase rotated symbol transmitted from antenna $l_{a}^{(p)}$ is denoted by $\grave{x}_{l_{a}^{(p)}}$. Accordingly, at time instant $k$, using the $N_{t}$-dimensional standard basis vector $\mathbf{e}_{n}$ (i.e., $\mathbf{e}_{1}=[1,0, \ldots, 0]^{T}$ ), the $N_{t}$-dimensional transmit vector $\mathbf{x}_{k}^{(p)} \in \mathbb{C}^{N_{t} \times 1}$ is expressed as

$$
\mathbf{x}_{k}^{(p)}=\left[\begin{array}{c}
\mathbf{0}_{\left(l_{a}^{(p)}-1\right) \times 1, k} \\
\grave{x}_{l_{a}^{(p)}, k} \\
\mathbf{0}_{\left(N_{t}-l_{a}^{(p)}\right) \times 1, k}
\end{array}\right]
$$

where $\mathbf{0}_{q \times p}$ is a $q \times p$ all-zero matrix. Therefore, the transmitted codeword is expressed as

$$
\mathbf{X}^{(p)}=\left[\begin{array}{ccc}
\mathbf{0}_{\left(l_{a}^{(p)}-1\right) \times 1,1} & \ldots & \mathbf{0}_{\left(l_{a}^{(p)}-1\right) \times 1, K} \\
\grave{x}_{l_{a}^{(p)}, 1} & \cdots & \grave{x}_{l_{a}^{(p)}, K} \\
\mathbf{0}_{\left(N_{t}-l_{a}^{(p)}\right) \times 1,1} & \ldots & \mathbf{0}_{\left(N_{t}-l_{a}^{(p)}\right) \times 1, K}
\end{array}\right] .
$$

GSTSM has the capability to adapt various numbers of transmitting antennae and constellation sizes, as well as a high granularity of phase rotations. In Section IV, it is also shown that an arbitrarily large number of receiving antennae can be used to improve the detection reliability of both of the information subchannels. Since we focus on systems with two transmit antennas, two phase rotations, and BPSK modulation, for a block length of $K, P=2^{K}$ patterns are available and therefore $\log _{2}(P)=K$-bit $I_{p}$ can be transmitted per block with an appropriate mapping. In the following GSTSM encoding example, we assume a GSTSM scheme with $K=6, n_{p}=2, \mathcal{O}=2, N_{t}=2$, and BPSK conventionally modulated symbols as shown in Fig. 1. The $I_{p}$ and $I_{c}$ bits are "10" and "110010", respectively. First, the $I_{c}$ stream is mapped onto BPSK symbols as $\mathbf{X}=\left[\begin{array}{llllll}-1 & -1 & 1 & 1 & -1 & 1\end{array}\right]$. Each possible $I_{p}$ stream is then mapped to a pattern and, subsequently, to a characteristic vector. The following mapping can be considered.

$$
\begin{aligned}
& \text { "00" } \rightarrow p=1 \rightarrow \boldsymbol{\zeta}^{(1)}=\left(\boldsymbol{\Lambda}^{(1)}, \boldsymbol{\xi}^{(1)}\right)=\left[\begin{array}{llllll}
1 & 1 & 1 & 1 & 1 & 1 \\
0 & 0 & 0 & 0 & 0 & 0
\end{array}\right] \\
& " 01 " \rightarrow p=2 \rightarrow \boldsymbol{\zeta}^{(2)}=\left(\boldsymbol{\Lambda}^{(2)}, \boldsymbol{\xi}^{(2)}\right)=\left[\begin{array}{llllll}
1 & 0 & 1 & 0 & 1 & 0 \\
0 & 2 & 0 & 2 & 0 & 2
\end{array}\right] \\
& " 11 " \rightarrow p=3 \rightarrow \boldsymbol{\zeta}^{(3)}=\left(\boldsymbol{\Lambda}^{(3)}, \boldsymbol{\xi}^{(3)}\right)=\left[\begin{array}{llllll}
0 & 0 & 0 & 0 & 0 & 0 \\
2 & 2 & 2 & 2 & 2 & 2
\end{array}\right] \\
& " 10 " \rightarrow p=4 \rightarrow \boldsymbol{\zeta}^{(4)}=\left(\boldsymbol{\Lambda}^{(4)}, \boldsymbol{\xi}^{(4)}\right)=\left[\begin{array}{llllll}
0 & 1 & 0 & 1 & 0 & 1 \\
2 & 0 & 2 & 0 & 2 & 0
\end{array}\right]
\end{aligned}
$$

The selected characteristic pattern vectors are used to specify the phase rotations as well as the active antennas for the transmission of the conventionally modulated symbols $\mathbf{X}$. The $I_{p}$ is " 10 ", and therefore the $p=4$ is chosen. Hence, the phase rotations are calculated from $\psi_{4, k}=\exp \left\{j \frac{\pi}{2}\left(\boldsymbol{\xi}_{k}^{(4)}-1\right)\right\}$ as $\psi_{4,1}=j, \psi_{4,2}=1, \psi_{4,3}=j, \psi_{4,4}=1, \psi_{4,5}=j$, and $\psi_{4,6}=1$. Also, according to the characteristic antenna activation vector $\Lambda^{(4)}$, the activated transmit antenna indices from time instant one to six are $l_{a}^{(4)}=[2,1,2,1,2,1]$. Hence, the transmitted GSTSM word is given by

$$
\mathbf{X}^{(4)}=\left[\begin{array}{cccccc}
0 & -1 & 0 & 1 & 0 & 1 \\
-j & 0 & j & 0 & -j & 0
\end{array}\right]
$$

In this work, the patterns are constructed based on the same principle as in STSM [9]. However, in GSTSM, each pattern is mapped onto both spatial and temporal dimensions of the system. Therefore, for a specific pattern $p$, a similar mapping is used for each dimension according to the method that has been introduced in [9].

\section{B. GSTSM Receiver Processing}

For a MIMO uplink system with $N_{t}$ transmitting antennae and $N_{r}$ receiving antennae, the received signal is given by

$$
\mathbf{Y}=\mathbf{H X}^{(p)}+\mathbf{N}
$$

where $\mathbf{H}$ is the $N_{r} \times N_{t}$ MIMO channel matrix and $\mathbf{N}$ is the $N_{r} \times K$ noise matrix consisting of independent and identically distributed (i.i.d.), zero-mean, complex Gaussian samples with variance $2 \sigma_{n}^{2}$. To find the transmitted word, the maximumlikelihood (ML) detector is expressed as

$$
\hat{\mathbf{X}}^{(p)}=\arg \min _{\mathbf{X}^{(p)} \in \mathcal{V}}\left\{\digamma\left(\mathbf{X}^{(p)}\right)\right\}
$$

where

$$
\digamma\left(\mathbf{X}^{(p)}\right)=\left\|\mathbf{Y}-\mathbf{H X}^{(p)}\right\|^{2}
$$

and $\mathcal{V}$ is the set of all possible transmitted codewords. The minimization in (5) involves exhaustive calculations over all possible codewords (i.e., symbols and patterns), which is prohibitively complex to be performed at the receiver side. Hence, using algebraic manipulations, the Euclidean cost function $\digamma\left(\mathbf{X}^{(p)}\right)$ can be calculated as

$$
\digamma\left(\mathbf{X}^{(p)}\right)=\sum_{k=1}^{K}\left\|\mathbf{Y}_{k}-\mathbf{H} \mathbf{X}_{k}^{(p)}\right\|^{2} .
$$

Since in the summation of (6) the terms are independent of each other, the overall minimization can be obtained via the minimization of each term. Consequently, the estimation of conventionally modulated symbols that minimizes the Euclidean cost $\digamma\left(\mathbf{X}^{(p)}\right)$ can be formulated as

$$
\hat{\mathbf{X}}_{k}^{(p)}=\arg \min _{\mathbf{X}_{k}^{(p)} \in \chi}\left\|\mathbf{Y}_{k}-\mathbf{H} \mathbf{X}_{k}^{(p)}\right\|^{2}, \forall k=1, \ldots, K
$$

where $\chi$ denotes the set of all legitimate transmitted vectors based on the available patterns. Therefore, for a specific pattern $p$, the corresponding Euclidean cost function is expressed as

$$
\begin{aligned}
\digamma_{\min }(p) & =\min \left\{\sum_{k=1}^{K}\left\|\mathbf{Y}_{k}-\mathbf{H} \mathbf{X}_{k}^{(p)}\right\|^{2}\right\} \\
& =\sum_{k=1}^{K}\left\|\mathbf{Y}_{k}-\mathbf{H} \hat{\mathbf{X}}_{k}^{(p)}\right\|^{2}
\end{aligned}
$$


After estimating the symbols using (7), the Euclidean cost (8) can be calculated for each of the possible patterns. Denoting the set of all patterns by $\mathcal{P}$, the ML solution is given by

$$
\hat{p}=\arg \min _{p \in \mathcal{P}}\left\{\digamma_{\min }(p)\right\} .
$$

The norm calculation in (7) requires $2|\mathcal{M}| N_{r}$ complex multiplications for each $k=1, \ldots, K$. The calculated Euclidean costs can be then used in the summations of (8) for calculating the Euclidean cost of each pattern. For each $k$, the Euclidean cost in (7) can take as many values as the number of supermodulation states $\mathcal{O}_{s m}$. Therefore, the complexity of ML detection is $2|\mathcal{M}| N_{r} K \mathcal{O}_{s m}$ complex multiplications. For the simulation evaluations of Section IV, $\mathcal{O}_{s m}=2$ is assumed, so the complexity is manageable.

\section{Headerless Grant-Free Multiple AcCess}

In this section, we discuss how GSTSM enables headerless and uncoordinated one-shot transmission for MTCs, and without the need for communicating any scheduling request. The resulting throughput gains versus traditional header-based schemes are presented in Section IV. The scenario considered here assumes that multiple machines communicate with the access point (AP) in the uplink, and that the AP employs one or more receive antennae. The data payload is ratelessly encoded, modulated, and transmitted in sets of blocks of size $K$ symbols, similarly to the system model in [8][9]. Each machine continuously transmits coded information blocks and the received information is detected, combined, and decoded at the AP. This process continues until the machine receives acknowledgement (ACK) from the AP that confirms that the related data payload has been successfully decoded. Using a dedicated channel, these ACK messages are broadcasted from AP and it is assumed that they are perfectly received. While the proposed grant-free multiple access scheme is based on rateless codes, the application of GSTSM is transparent to the choice of the channel coding method and it can be used in conjunction with other schemes that detect and combine the received information [19]. Also, the proposed approach is applicable to other wireless networks such as ad-hoc MTCs, as well as to scenarios with random data transmission.

In order to combine and decode the received information blocks at the AP, the receiver needs to know the identity of transmitting machine, as well as the relative position of the received packet in the whole rateless coded packet sequence. Hence, additional information bits need to be sent to carry this information, which is referred to as signature information (SI). Here, the SI consists of two parts. The first, consisting of $k_{u}$ bits, is used for providing the identity of the machine. The second, consisting of $k_{o}$ bits, provides the order of the packet in the rateless coded packet sequence. To transmit each or all segments of the SI, several methods can be employed. The first approach is based on the mapping of SI bits to orthogonal sequences such as the Zadoff-Chu (ZC), that are used in RACH of LTE [20]. Then, the SI is transmitted by means of preambles/headers that are sent along with the coded packets. Instead of mapping the SI onto orthogonal sequences, it can be encoded by means of strong, low-rate channel codes [6] and transmitted as a packet header.

Whether the SI is transmitted via mapping to $\mathrm{ZC}$ sequences or via using low-rate channel codes, both methods require the transmission of a packet header that can considerably limit the achievable throughput of the system. Instead, by supermodulating the SI, we obviate the need for packet headers, resulting in significant throughput gains as shown in Section IV. For the rateless encoding of the data payload, Raptor codes [21] have been used since they are among the most widely used rateless schemes due to their low-complexity decoding. The encoding and decoding of Raptor codes are described in [21]. For decoding, the soft-information-based belief-propagation decoder is used. The soft information of the conventionally modulated bits can be calculated as

$$
L_{\left(x_{i, q}\right)}=\ln \left(\frac{\sum_{x_{i, q} \in \mathcal{W}_{q}^{0}} \sum_{l \in N_{t}} \sum_{\psi_{i} \in \Phi_{l}} \exp \left[\frac{-\left\|\mathbf{Y}_{i}-\mathbf{h}_{l} \psi_{i} x_{i, q}\right\|^{2}}{2 \sigma_{n}^{2}}\right]}{\sum_{x_{i, q} \in \mathcal{W}_{q}^{1}} \sum_{l \in N_{t}} \sum_{\psi_{i} \in \Phi_{l}} \exp \left[\frac{-\left\|\mathbf{Y}_{i}-\mathbf{h}_{l} \psi_{i} x_{i, q}\right\|^{2}}{2 \sigma_{n}^{2}}\right]}\right)
$$

where $L_{\left(x_{i, q}\right)}$ denotes the Log-Likelihood Ratio (LLR) of $q^{\text {th }}$ bit of the conventional symbol $x_{i}, \mathbf{h}_{l}$ denotes the $N_{r} \times 1$ vector with the channel coefficient(s) from the transmitting antenna $l$ to the receiving antennae, $\Phi_{l}$ denotes the set of available phase rotations for the transmitter antenna $l$, and $\mathcal{W}^{0}$ and $\mathcal{W}^{1}$ are the subsets of the conventional symbols that have the $q^{\text {th }}$ bit equal to 0 and 1 , respectively.

\section{Simulation Evaluations}

In this section, GSTSM's performance is evaluated via simulations. It is assumed that each machine employs two transmitting antennae (with the transmitted power normalized to unity), and that the conventional information is BPSK modulated. The MIMO channel is modeled as a temporally and spatially uncorrelated frequency-flat Rayleigh channel, unless stated otherwise. Also, it is assumed that perfect knowledge of the channel is available at the receiver. Fig. 2 shows the uncoded bit-error-rate (BER) performance for both the subchannels $I_{c}$ and $I_{p}$, and for several sizes of super-modulated bit sequences $n_{p}$, resulting in a corresponding throughput increase of $T_{g}$. It is shown that the reliability of $I_{p}$ is significantly higher than that of $I_{c}$. It is also shown that the BER for $I_{p}$ is better for a smaller $n_{p}$, while the performance of $I_{c}$ remains unaffected even for a large value of $n_{p}$. Hence, a throughput gain of $10 \%$ can be obtained without affecting the BER of $I_{c}$. In addition, it can be observed that the reliability of both information subchannels is substantially enhanced for large MIMO systems. For example, to attain throughput gain of $10 \%$ at BER of $10^{-4}$, using $N_{r}=10$. Specifically for the super-modulated bits, no error has been detected after transmitting $10^{5}$ information packets. It can be observed that a highly-reliable information stream with a flexible-rate is provided by GSTSM. This cannot be achieved with traditional spatial modulation schemes. 


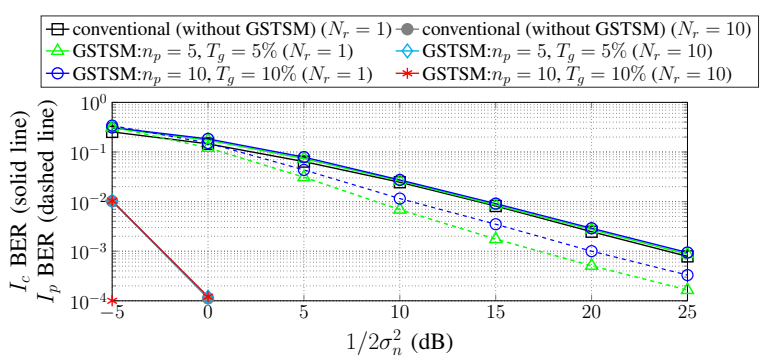

Fig. 2: The BER performance of the individual subchannels of GSTSM for several $n_{p}$ values (yielding a throughput gain of $T_{g}$ ) versus traditional scheme (without GSTSM) for $K=100$. For $I_{p}$ with $n_{p}=5$ and $N_{r}=10$, no error has been observed when transmitting $10^{5}$ information packets.

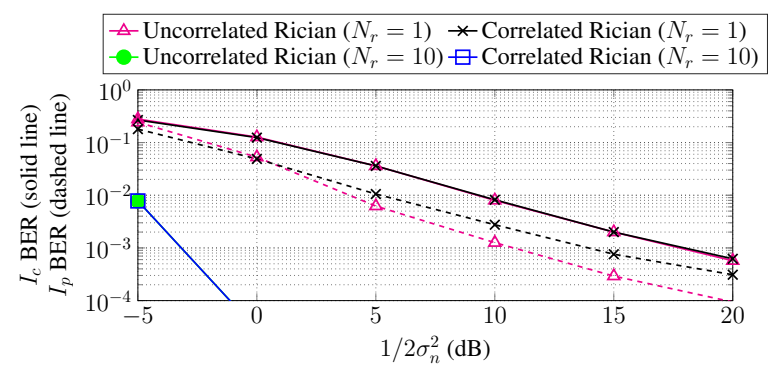

Fig. 3: Comparison of the BER performance of the individual subchannels of GSTSM for correlated and uncorrelated Rician fading channels with Rician $K_{\text {Ric. }}=3$ factor, and for $n_{p}=5$ and $K=100$. For $I_{p}$ with $N_{r}=10$, no error has been observed when transmitting $10^{5}$ information packets.

Fig. 3 depicts the BER performance of systems as in previous figure with $n_{p}=5$, but in highly correlated channel conditions with a strong Rician $K_{R i c} .=3$ factor which is in the range of values measured in indoor communications [17]. Since in such highly correlated channel conditions there is no significant difference between the propagation characteristics of the transmitting antennae, GSTSM (similarly to spatial modulation) cannot rely on the spatial dimension to detect the transmitted pattern. However, Fig. 3 shows that the supermodulated bits can still be detected with small performance degradation. This is achieved since GSTSM also exploits the temporal dimension of the transmitted signal. Again, the large antenna numbers at the receiver side, result in a substantially improved reliability for both super-modulated and conventional information subchannels. In Fig. 4, the performance of GSTSM is evaluated in a single-input multipleoutput (SIMO) system over an Additive White Gaussian Noise (AWGN) channel. It is shown that even when the spatial dimension is not explored, GSTSM still enables a reliable transmission of both information subchannels, just based on the introduced temporal correlation (i.e., phase rotations). The detection reliability can again be substantially improved by exploiting multiple receive antennae and receive diversity.

Fig. 5 (a) presents the achievable rate of a ratelessly coded system for one user with a payload size of 1000 bits, in a collision-free environment. Different approaches are examined for the transmission of each packet's SI. A $k_{s i}=9$-bit SI consisting of $k_{u}=6$ bits (to support 64 users as in LTE

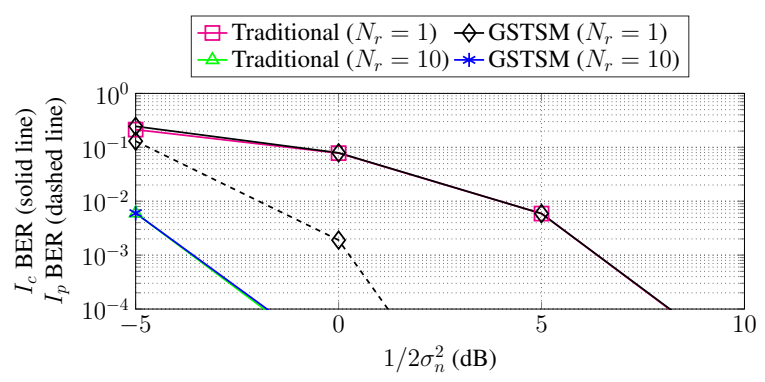

Fig. 4: Comparison of the BER performance of the individual subchannels of GSTSM with $n_{p}=5, K=100$, and for an AWGN channel. For $I_{p}$ with $N_{r}=10$, no error has been observed after transmitting $10^{5}$ information packets.

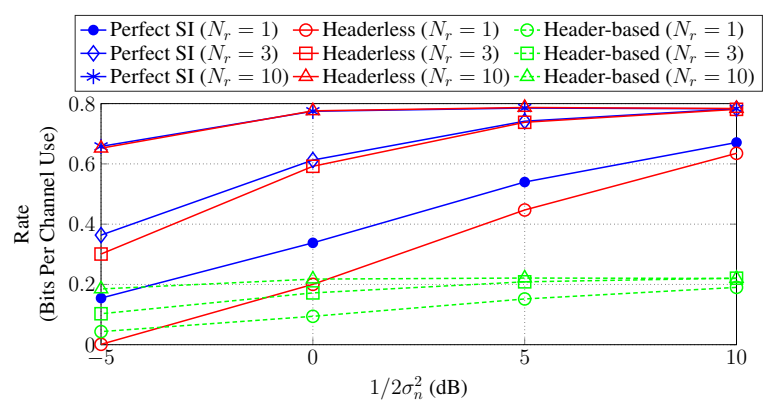

(a)

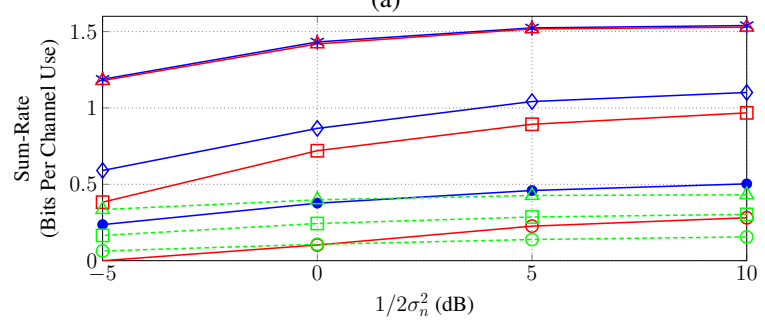

(b)

Fig. 5: Comparison of the achievable rate of the rateless systems employing headerless (GSTSM) and header-based (ZC) schemes for $K=200$ and for a varying number of receive antennae: (a) rate in multiuser collision-free environment (b) sum-rate in multiuser environments with two colliding users.

$\mathrm{RACH})$ and $k_{o}=3$ bits is used. To transmit $k_{s i}$ bits using ZC sequences, it is required to map them to $2^{k_{s i}}$ sequences with a minimum length of $2^{k_{s i}}$ samples. Therefore, $\mathrm{ZC}$ sequences with a minimum of 512 samples are required for conveying $k_{s i}=9$-bit SI. We have compared the performance of ZC and channel coded preambles (i.e., convolutional coded as in LTE control channels), and we observed that $\mathrm{ZC}$ preambles have superior performance. Hence, we only focus on ZC preambles. The following three cases are considered for delivering $k_{s i}=$ 9 -bit SI. The case where SI is perfectly known, the case where all SI bits are transmitted using GSTSM (i.e., headerless), and the case where all SI bits are mapped onto ZC sequences (i.e., header-based). Raptor rateless codes have been used to encode each packet, which are constructed based on an inner LT code according to Raptor RFC 5053 [22], and a rate 0.95 LDPC precode with left regular distribution (degree 3 nodes) and right Poisson. For decoding, the sum-product algorithm [21] 


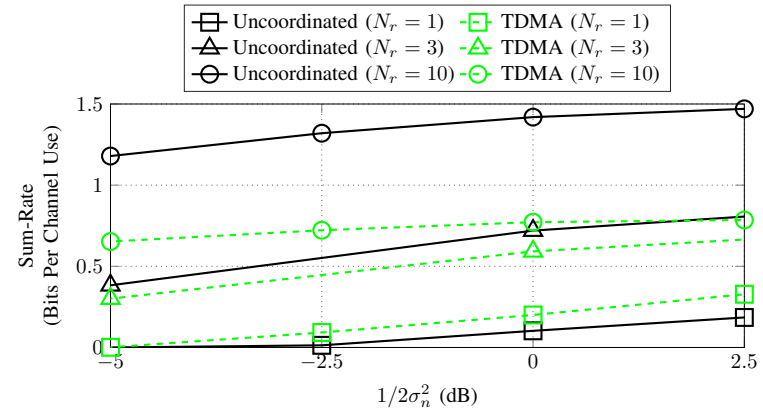

Fig. 6: Comparison of the sum-rate of the GSTSM-enabled rateless systems with two users and $K=200$, based on uncoordinated access and TDMA.

is executed with forty iterations. In all cases, it is assumed that if a signature information packet has been found more than once, the most reliable packet is selected according to their soft metrics. Also, when the number of transmitted packets exceeds the number that can be counted by $k_{o}$, the counter resets. As shown in Fig. 5 (a), by using $N_{r}=1$, a GSTSMenabled scheme approaches the ideal case and outperforms the header-based approach for a broad range of SNRs. In the case of large $N_{r}$, GSTSM yields a performance much closer to the ideal case while also providing superior performance compared to the header-based cases in the whole SNR range, enabling efficient headerless transmission.

In addition, since in grant-free-based MTCs packet collisions are likely to happen, the appropriateness of GSTSM is also examined for the extreme case of uncoordinated transmission. In this case, it is assumed that the transmitted packets of two machines are always colliding, similar to the two-user multiple access channel [23]. The attainable sum-rate for the various cases is shown in Fig. 5 (b). It is shown that GSTSM can result in up to $2.5 \times$ higher throughput compared to headerbased approach, across the whole SNR range.

Fig. 6 compares the achievable sum-rate of a GSTSMenabled scheme for two machines, for uncoordinated (colliding) and collision-free (i.e., TDMA) transmissions. It is shown that coordinated transmissions yield superior performance when just a single antenna is used at the receiver. However, due to GSTSM, the rateless properties of the transmitted information can be also exploited, and the uncoordinated scheme can yield significantly higher throughput gains, especially when the number of receiving antennae is increased. Hence, by using $N_{r}=10$, throughput gains of up to $85 \%$ can be attained compared to the TDMA case, where only one machine is transmitting at each time instant.

\section{CONCLUSIONS}

We have introduced the concept of Generalized Space-Time Super-Modulation that enables uncoordinated, grant-free, and headerless rateless transmissions for machine-type communications. It is shown that GSTSM results in throughput gains that approach the theoretical gains of multiple access channels, while it also obviates the need for centralized coordination as in TDMA-based systems.

\section{ACKNOWLEDGMENT}

The authors would like to thank the members of University of Surrey 5GIC (http://www.surrey.ac.uk/5GIC) for their support.

\section{REFERENCES}

[1] S. Chen, et al., "Machine-to-machine communications in ultra-dense networks - a survey," IEEE Commun. Surveys Tuts., vol. 19, no. 3, pp. 1478-1503, Third Quarter 2017.

[2] N. Xia, et al., "Radio resource management in machine-to-machine communications - a survey," IEEE Commun. Surveys Tuts., vol. 20, no. 1, pp. 791-828, First Quarter 2018.

[3] Y. D. Beyene, et al., "Random access scheme for sporadic users in 5G," IEEE Trans. Wireless Commun., vol. 16, no. 3, pp. 1823-1833, Mar. 2017.

[4] H. Shariatmadari, et al., "Machine-type communications: current status and future perspectives toward 5G systems," IEEE Commun. Mag., vol. 53, no. 9, pp. 10-17, Sep. 2015.

[5] C. Bockelmann, et al., "Massive machine-type communications in 5G: physical and MAC-layer solutions," IEEE Commun. Mag., vol. 54, no. 9, pp. 59-65, Sep. 2016.

[6] M. Wang, et al., "The evolution of LTE physical layer control channels," IEEE Commun. Surveys Tuts., vol. 18, no. 2, pp. 1336-1354, Second Quarter 2016.

[7] F. Mehran, K. Nikitopoulos, et al., "Rateless wireless systems: gains, approaches, and challenges," in Proc. 3rd IEEE ChinaSIP, 2015.

[8] K. Nikitopoulos, F. Mehran, and H. Jafarkhani, "Space-time supermodulation and its application to joint medium access and rateless transmission," in Proc. IEEE GLOBECOM 2016.

[9] K. Nikitopoulos, F. Mehran, and H. Jafarkhani, "Space-time supermodulation: concept, design rules, and its application to joint medium access and rateless transmission," IEEE Trans. Wireless Commun., vol. 16, no. 12, pp. 8275-8288, Dec. 2017.

[10] S. M. Alamouti, "A simple transmit diversity technique for wireless communications," IEEE J. Sel. Areas Commun., vol. 16, no. 8, pp. 14511458, Oct. 1998.

[11] R. Y. Mesleh, et al., "Spatial modulation," IEEE Trans. Veh. Technol, vol. 57, no. 4, pp. 2228-2241, Jul. 2008.

[12] S. Yang, et al., "Fifty years of MIMO detection: the road to large-scale MIMOs," IEEE Commun. Surveys Tuts., vol. 17, no. 4, pp. 1941-1988, Fourth Quarter 2015.

[13] P. Yang, et al., "Design guidelines for spatial modulation," IEEE Commun. Surveys Tuts., vol. 17, no. 1, pp. 6-26, First Quarter 2015.

[14] U. Wachsmann, et al., "Multilevel codes: theoretical concepts and practical design rules," IEEE Trans. Inf. Theory, vol. 45, no. 5, pp. 13611391, Jul. 1999.

[15] H. Imai, et al., "A new multilevel coding method using error correcting codes," IEEE Trans. Inf. Theory, vol. 23, no. 3, pp. 371-377, May 1977.

[16] G. Ungerboeck, "Trellis coded modulation with redundant signal sets, part I: introduction," IEEE Commun. Mag., vol. 25, no. 2, pp. 5-11, Feb. 1987.

[17] R. Mesleh, et al., "Trellis coded spatial modulation," IEEE Trans. Wireless Commun., vol. 9, no. 7, pp. 2349-2361, Jul. 2010.

[18] G. Georgis, et al., "Geosphere: an exact depth-first sphere decoder architecture scalable to very dense constellations," IEEE Access, vol. 5, pp. 4233-4249, Mar. 2017.

[19] H. Zhuang, et al., "Flexible HARQ: a high throughput hybrid-ARQ protocol," in Proc. IEEE ICC 2017.

[20] H. S. Jang, et al., "Message-embedded random access for cellular M2M communications," IEEE Commun. Lett., vol. 20, no. 5, pp. 902-905, May 2016.

[21] O. Etesami, et al., "Raptor codes on binary memoryless symmetric channels," IEEE Trans. Inf. Theory, vol. 52, no. 5, pp. 2033-2051, May 2006.

[22] M. Luby, et al., "Raptor forward error correction scheme for object delivery," RFC 5053, Oct. 2007.

[23] D. Tse and P. Viswanath, Fundamentals of Wireless Communication, Cambridge, UK: Cambridge University Press, 2005. 